\title{
TOURnament versus Fitness Uniform Selection
}

\author{
Shane Legg \\ IDSIA \\ Galleria 2, CH-6928, \\ Manno-Lugano, Switzerland \\ Email: shane@idsia.ch
}

\author{
Marcus Hutter \\ IDSIA \\ Galleria 2, CH-6928, \\ Manno-Lugano, Switzerland \\ Email: marcus@idsia.ch
}

\author{
Akshat Kumar \\ Indian Institute of Technology \\ Guwahati \\ India \\ Email: akshat@iitg.ernet.in
}

\begin{abstract}
${ }^{1}$ In evolutionary algorithms a critical parameter that must be tuned is that of selection pressure. If it is set too low then the rate of convergence towards the optimum is likely to be slow. Alternatively if the selection pressure is set too high the system is likely to become stuck in a local optimum due to a loss of diversity in the population. The recent Fitness Uniform Selection Scheme (FUSS) is a conceptually simple but somewhat radical approach to addressing this problem - rather than biasing the selection towards higher fitness, FUSS biases selection towards sparsely populated fitness levels. In this paper we compare the relative performance of FUSS with the well known tournament selection scheme on a range of problems.
\end{abstract}

\section{Introduction}

The standard selection schemes used in evolutionary algorithms (such as tournament, ranking, proportional, truncation selection, and so on) all focus the selection pressure towards individuals of higher fitness in the population. The rational being that these individuals are the most likely to produce offspring (either by mutation or crossover or both) that belong to still higher fitness levels. For many problems this is often a valid assumption, however for difficult, deceptive and highly multi-modal functions the path towards the global optimum is rarely smooth (by "deceptive" we mean in the general rather than the technical sense). In these cases it is important that we explore the solution space very carefully before becoming too committed to any subset of solutions that appears to be promising. In order to do this we must not focus too much of our search energy on only the most fit individuals. In particular we must ensure that we keep some less fit individuals in the population in case we need to use them to initialize a new direction of exploration should we become stuck in a local optimum.

For standard selection schemes this is controlled by appropriately setting the parameters that govern the selection pressure on the individuals. If this pressure is set too high the evolutionary algorithm (EA) will

\footnotetext{
${ }^{1}$ This work was supported by SNF grant 2100-67712.02.
}

converge quickly but possibly to a local optimum, while if it is set too low the system will converge only very slowly, if at all. It is often the case that this can only be done through a process of experimentation with the particular problem at hand.

Many systems have been devised to help prevent this problem by ensuring that the population maintains a certain degree of diversity. Significant contributions in this direction are fitness sharing [1, crowding [2] and local mating 3. The Fitness Uniform Selection Scheme (FUSS) [4] is another proposed solution to this problem which is well suited for very difficult optimization problems. The key idea is to preserve genetic diversity in the population by using the fitness of individuals to estimate their similarity. The beauty of this approach is that it is simple to implement, problem intrinsic and also representation independent.

In this paper we present the first experimental investigations into the performance of FUSS. Our goal is to develop a better understanding of its performance characteristics in practice and in particular how it compares to a standard selection scheme which favors fit individuals.

Section 2 briefly explains how the relative fitness of individuals can be used to define a simple metric that provides us with some indication of the similarity of individuals. In Section 3 we discuss the definition of the fitness uniform selection scheme for both discrete and continuous valued fitness functions. We also outline some of its key theoretical properties and contrast these with standard selection schemes. Section 4 details our experimental setup. Section $[5$ examines the performance of FUSS and tournament selection on an artificially constructed deceptive optimization problem. We compare our results to the behavior predicted in [4. The performance of FUSS and tournament selection is then examined on a set of randomly generated integer valued functions in Section [ [n Section 7 we detail the performance of FUSS and tournament selection on both artificial and real traveling salesman problems. In Section 8 we examine the set covering problem, an NP hard optimization problem which has many real world applications. For our final test in Section 9 we compare FUSS and tournament selection on random maximum CNF3 SAT problems and graph coloring problems which have been expressed in the CNF 

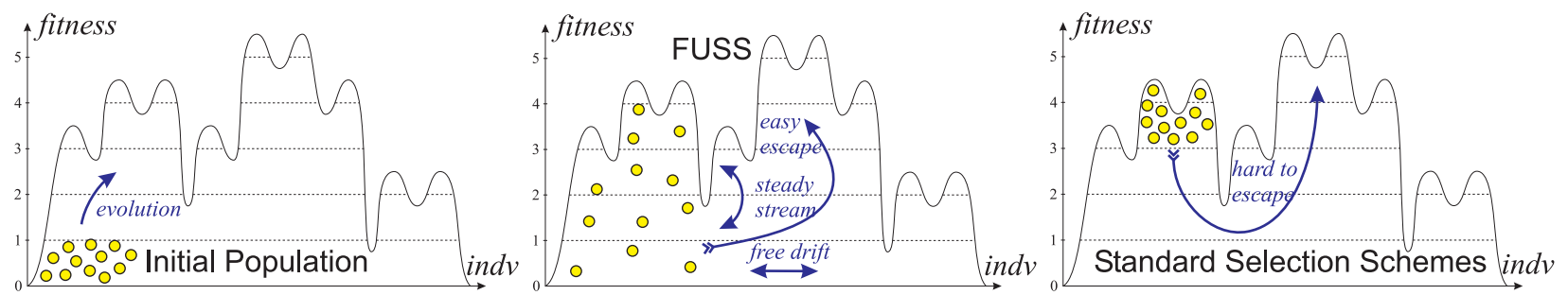

Figure 1: Evolution of the population under FUSS versus standard selection schemes (STD): STD may get stuck in a local optimum if all unfit individuals were eliminated too quickly. In FUSS, all fitness levels remain occupied with "free" drift within and in-between fitness levels, from which new mutants are steadily created, occasionally leading to further evolution in a more promising direction.

form. These are also NP hard optimization problems. Section 10 contains a brief summary of our results and possible avenues for future research.

\section{Using Fitness to Measure Sim- ilarity}

There are many ways to measure the similarity of individuals in a population. If the individuals are binary coded one might use the Hamming distance as a similarity relation. This distance is consistent with a mutation operator which flips a few bits. It produces Hamming-similar individuals, but recombination (like crossover) can produce very dissimilar individuals w.r.t. this measure. In any case, genotypic similarity relations, like the Hamming distance, depend on the representation of the individuals as binary strings. Individuals with very dissimilar genomes might actually be functionally (phenotypically) very similar. For instance, when most bits are unused (like introns in GP), they can be randomly disturbed without affecting the property of the individual. For specific problems at hand, it might be possible to find suitable representation-independent functional similarity relations. On the other hand, in genetic programming, for instance, it is in general undecidable, whether two individuals are functionally similar.

FUSS takes a different approach. The distance between two individuals $i$ and $j$ with fitness $f(i)$ and $f(j)$ is defined as

$$
d(i, j):=|f(i)-f(j)|
$$

The distance is based solely on the fitness function, which is provided as part of the problem specification. It is independent of the coding/representation and other problem details, and of the optimization algorithm (e.g. the genetic mutation and recombination operators), and can trivially be computed from the fitness values. If we make the natural assumption that functionally similar individuals have similar fitness, they are also similar w.r.t. the distance $d$. On the other hand, individuals with very different coding, and even functionally dissimilar individuals may be $d$-similar, but we will see that this does not matter.

For instance, individuals from different local optima of equal height are $d$-similar.

Armed with this simple measure of similarity between individuals we can now define a selection scheme that aims to preserve diversity in the population.

\section{Fitness Uniform Selection Strategy (FUSS)}

The idea behind FUSS is that we should focus the selection pressure towards fitness levels which have relatively few individuals rather than on the highest fitness levels. In this way fitness levels which are difficult to reach are thoroughly explored and on no fitness level does the population size decrease towards extinction (see Figure 11). Thus FUSS preserves genetic diversity more actively than the standard selection schemes which tend to drive the populations on lower fitness levels to zero. Moreover, parts of the fitness space which are interesting, in the sense that they are difficult to reach, are focused on, rather than easy to reach areas which are already well represented in the population. This approach might seem counter intuitive as we are not even attempting to increase the average fitness of the population! The point is that for optimization problems we are usually only interested in finding a single individual with the highest possible fitness having low average fitness is not in itself a problem.

For general real-valued fitness functions FUSS is defined as follows: A uniform random number is chosen in the interval $\left[f_{\min }, f_{\max }\right]$, where $f_{\max }$ and $f_{\min }$ are the maximum and minimum fitness values in the current population. Then the individual with fitness nearest to this number is chosen (see Figure 2). If this is 


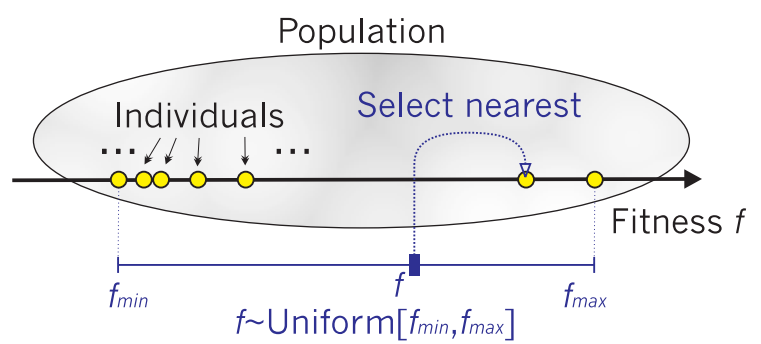

Figure 2: If the lowest/highest fitness values in the current population $P$ are $f_{\min / \max }$, FUSS selects a fitness value $f$ uniformly in the interval $\left[f_{\min }, f_{\max }\right]$, then, the individual $i \in P$ with fitness nearest to $f$ is selected and a copy is added to $P$, possibly after mutation and recombination.

ambiguous one of the nearest individuals is chosen at random. In the case of integer valued fitness functions this is equivalent to selecting a fitness level at random from the set $\left\{f_{\min }, f_{\min }+1, \ldots, f_{\max }\right\}$ and then randomly selecting an individual within that fitness level if the level is occupied. If the level is empty, higher and lower fitness levels are progressively searched until a non empty level is found at which time a random individual is selected.

While the probability of selecting each fitness level is equal, the probability of then selecting a given individual within a fitness level depends on the population of that level. For example, if an individual belongs to a fitness level with 50 members its selection probability is twice as high as an individual that belongs to a fitness level with 100 members. It is easy to see that under a selection scheme based on the FUSS approach, the proportion of individuals at each fitness level tends towards the fraction $\frac{1}{|F|}$, where $|F|$ is the number of fitness levels as depicted in Figure 3 . See 4 for a more detailed description.

While this preserves a greater degree of population diversity than the standard selection schemes, it comes at the cost of a potential loss of performance due to the large number of selections from low fitness levels. Thus the currently highest parts of the fitness space are now searched more slowly than under a standard selection scheme. In the worst case FUSS will slow the performance of the system down by a factor of $|F|$. However for significantly deceptive problems the loss of performance due to becoming stuck in a local optimum for a long period of time is a much more significant cost than a potential factor of $|F|$. It is for these problems that the author of 4 expects the strengths of FUSS to become evident.

At first glance it might appear that there is little pressure on selecting highly fit individuals under FUSS. Usually this is not the case as the most fit individuals
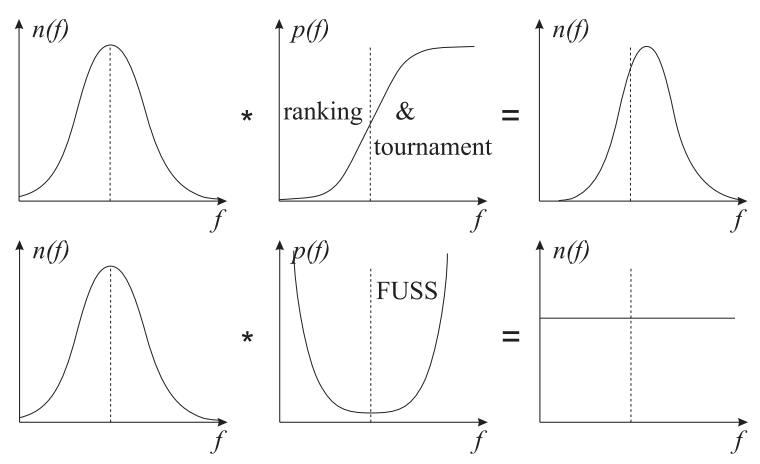

Figure 3: Effects of ranking=tournament and fitness uniform (FUSS) selection on the fitness distribution in a generation based EA. The left/right diagrams depict fitness distributions before/after applying the selection schemes depicted in the middle diagrams.

in a population are typically quite rare. If these individuals start to make up a significant proportion of the total population this indicates that this part of the space has been significantly searched and thus is more likely to be an evolutionary dead end. In this case FUSS will, by its nature, automatically move selection pressure away from these highly fit individuals and focus its search energy on lower fitness levels that have fewer individuals. In this way the selection intensity varies dynamically with the evolution of the population. Clearly this is preferable to the situation where we must manually fix the selection pressure for a particular optimization problem in order to prevent the system from becoming stuck in local optima.

\section{GA Test System}

We have implemented a GA test system in Java on a PC running Linux. The selection schemes include FUSS and the standard tournament selection scheme. With tournament selection we randomly pick a group of individuals and then select the fittest individual from this group. The size of the group is called the tournament size and it is clear that the larger this group is the more likely we are to select a highly fit individual from the population. A tournament size of 2 is commonly used in practice as this often provides sufficient selection intensity on the most fit individuals. In our tests we have used tournament sizes of 2, 5 and 15 which we will refer to as TOUR2, TOUR5 and TOUR15 respectively. This should provide some insight into how different levels of selection intensity affect performance in different problems.

We have chosen to compare FUSS with tournament selection as this scheme is simple to understand and im- 
plement and is also one of the most widely used. Also we consider it to be roughly representative of other standard selection schemes which favor the fitter individuals in the population; indeed in the case of tournament size 2 it can be show that tournament selection is equivalent to the linear ranking selection scheme [5], Sec.2.2.4]. At some point in the future we may implement other standard selection schemes to broaden our comparison, however we expect the performance of these schemes to be at best comparable to tournament selection when used with a correctly tuned selection intensity.

The GA model we have chosen is the so called "steady state" model as opposed to the more usual "generational" model. In a generational GA at each generation we select an entirely new population based on the old population. The old population is then simply discarded. Under the steady state model that we use, individuals are only selected one at a time: We select an individual, then with a certain probability we select another and cross the two to produce a new individual, and then with another probability we mutate the result. We will refer to the probability of crossing as the crossover probability and the probability of mutating following a cross as the mutate probability. In the case where no crossover took place the individual is always mutated to insure that we are not simply adding a clone of an existing individual into the population. Finally an individual must be deleted in order to keep the population size constant. How this is done is important as it can bias the population in a way that is similar to the selection scheme. We have chosen to simply delete a random individual from the population which is a common neutral strategy used in steady state GAs.

The number of generations in a generational GA is roughly equivalent to the number of iterations in a steady state GA divided by the population size. We have used this approximation here when reporting the number of generations on graphs etc. Unfortunately the theoretical understanding of the relationship between the two types of GA is quite poor. It has been shown that under the assumption of no crossover the effective selection intensity using tournament selection with size 2 is approximately twice as strong under a steady state GA as it is with a generational GA [6]. As far as we are aware a similar comparison for systems with crossover has not been performed, though we would not expect the results to be significantly different. While steady state GAs have certain advantages, the fact that generational GAs are more common means that we may in the future test FUSS under this model also.

The important free parameters to set for each test are the population size, and the crossover and mutation probabilities mentioned above. Our default is to have both the crossover and mutation probabilities set to 0.5. For each problem we conducted some preliminary experiments to establish reasonable settings for these variables. Often the effect of these variables on performance was not particularly strong, though it was always worth checking to be sure. More importantly, the relative performance of the selection schemes remained quite stable. For population sizes less than 500 performance tended to degrade for difficult problems where the potential solution space was large. To avoid this our experiments have been performed with populations of 1,000 individuals or more. For each test the parameters were the same for each selection scheme - indeed the only difference was which subroutine in the code was used to select individuals. This ensures that our comparison was fair.

In order to generate reliable statistics we ran each test multiple times; typically 20 or 30 times. From these runs we then calculated the average performance for each selection scheme. We also computed the sample standard deviation and from this the standard error in our estimate of the mean. This value was then used to generate the $95 \%$ confidence intervals which appear on the graphs.

\section{A Deceptive 2D Problem}

The first problem we examine is the simple but highly deceptive 2D problem for which the performance of FUSS was theoretically analyzed in [4]. The setup of the test is quite simple. The space of individuals is the unit square $[0,1] \times[0,1]$. On this space narrow regions $I_{1}:=[a, a+\delta] \times[0,1]$ and $I_{2}:=[0,1] \times[b, b+\delta]$ for some $a, b, \delta \in[0,1]$ are defined. Typically $\delta$ is chosen so that it is much smaller than 1 and thus $I_{1}$ and $I_{2}$ do not occupy much of the domain space. The fitness function is defined to be,

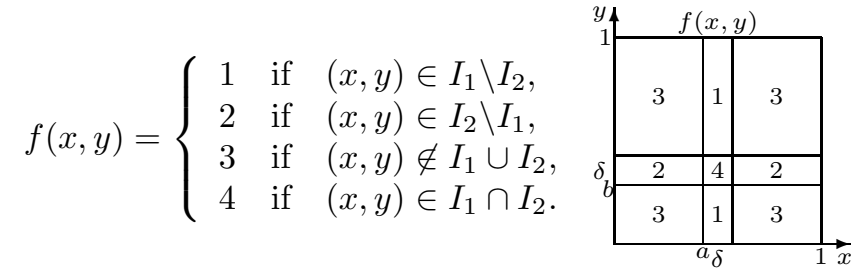

The example has sort of an XOR structure, which is hard for many optimizers.

For this problem we set up the mutation operator to randomly set either the $x$ or $y$ position of an individual and the crossover to take the $x$ position from one individual and the $y$ position from another to produce an offspring. 


\section{Deceptive 2D Problem}

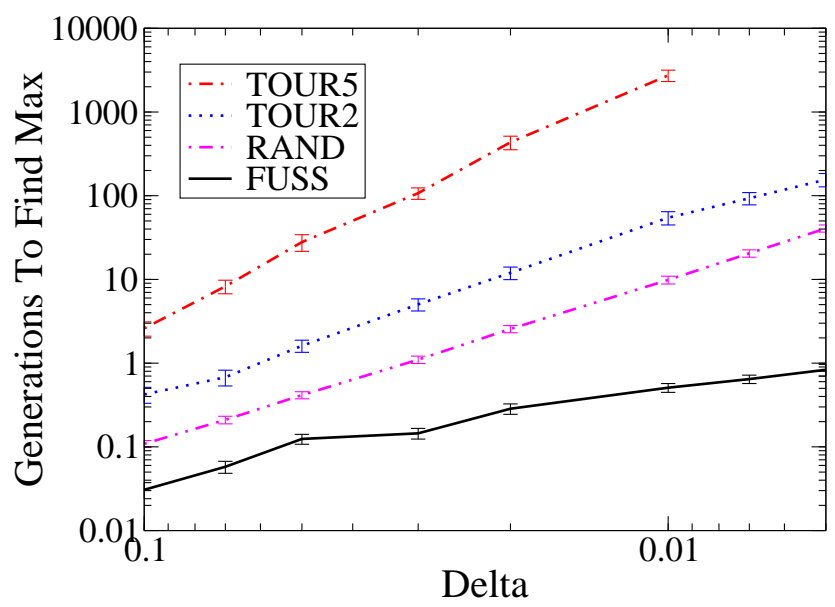

Figure 4: As predicted FUSS scales significantly better than both tournament selection and random search for this problem. Also, increasing selection pressure in tournament selection (TOUR2 vs. TOUR5) degraded performance.

Under these operators this is a very deceptive and difficult optimization problem. The size of the domain for which the function is maximized is just $\delta^{2}$ which is very small for small values of $\delta$. Moreover the local maxima at fitness level 3 covers most of the space and the only way to reach the global maximum is by leaving this local maxima and exploring the space of individuals with lower fitness value of 1 or 2 . For such a problem FUSS should in theory perform much better than either random search or more standard selection schemes.

For this test we set the maximum population size to 10,000 and ran each scheme for each delta value 20 times. With a steady state GA it is usual to start with a full population of random individuals. However for this particular problem we reduced the initial population size down to just 10 in order to avoid the effect of doing a large random search when we created the initial population and thereby distorting the scaling. Usually this might create difficulties due to the poor genetic diversity in the initial population. However due to the fact that any individual can mutate to any other in just two steps this is not a problem in this situation. Initial tests indicated that reducing the crossover probability from 0.5 to 0.25 improved the performance slightly and so we have used this setting. For comparison random search (RAND) was also implemented. The results of these tests appear in Figure 4

As expected higher selection pressure on the most fit individuals is clearly a disadvantage for this problem. With low selection pressure (TOUR2) tournament se- lection performs slightly worse than random search while with medium selection pressure (TOUR5) performance was in the order of 20 times slower than random search. With high selection pressure (TOUR15) the test became infeasible to compute. Our results confirm the theoretical scaling factors of $\frac{1}{\delta^{2}}$ for RAND and TOUR2, and $\frac{1}{\delta}$ for FUSS, as predicted in [4].

\section{Random Functions}

In order to gain a better understanding of how FUSS performs relative to tournament selection in more general problem settings we tested the selection schemes on a set of randomly generated functions. In this case the domain of each function was the 4 dimensional hyper cube $[0,1]^{4}$. To create each random function we randomly generated 16 cuboids of 4 dimensions inside the domain space. The function value of a point inside the domain space was then taken to be the number of random cuboids that contained the point. Thus, depending on where the random cuboids where, the range of the function could be anything from $\{0,1\}$ to $\{0,1, \ldots, 16\}$. This process of building up functions using cuboids allowed the functions to be quite complex and multi modal while still keeping some rough continuity. In order to make the optimization problem a little more tractable we limited the width of the cuboids in any one dimension to be in the range $[0.2,1]$. While this limited the minimum size of each random cuboid, two or more cuboids could still form arbitrarily small intersections and thus the domain region in which a function achieves its maximal value could still be extremely small.

In the first test we generated 100 random functions and precomputed each function's global maximum value by using the cuboid position information used to construct the function. For each function we then ran both FUSS and TOUR2 10 times and computed for each the average number of generations needed to find the global maximum. This produced 100 data points corresponding to the 100 random functions.

We first tested TOUR2 as we expected the problem to be relatively deceptive and thus higher selection pressure would be a disadvantage. The population size for these tests was set at 10,000. The results are plotted in Figure 15

We see that FUSS typically manages to find the global maximum 2 to 20 times faster than TOUR2, with 10 being about average. We then compared FUSS with TOUR5. The results of this test are plotted in Figure 5 .

Interestingly the performance of tournament selection improved to the extent that it was then roughly equivalent to FUSS. Typically a tournament size of 2 


\section{Random Functions}

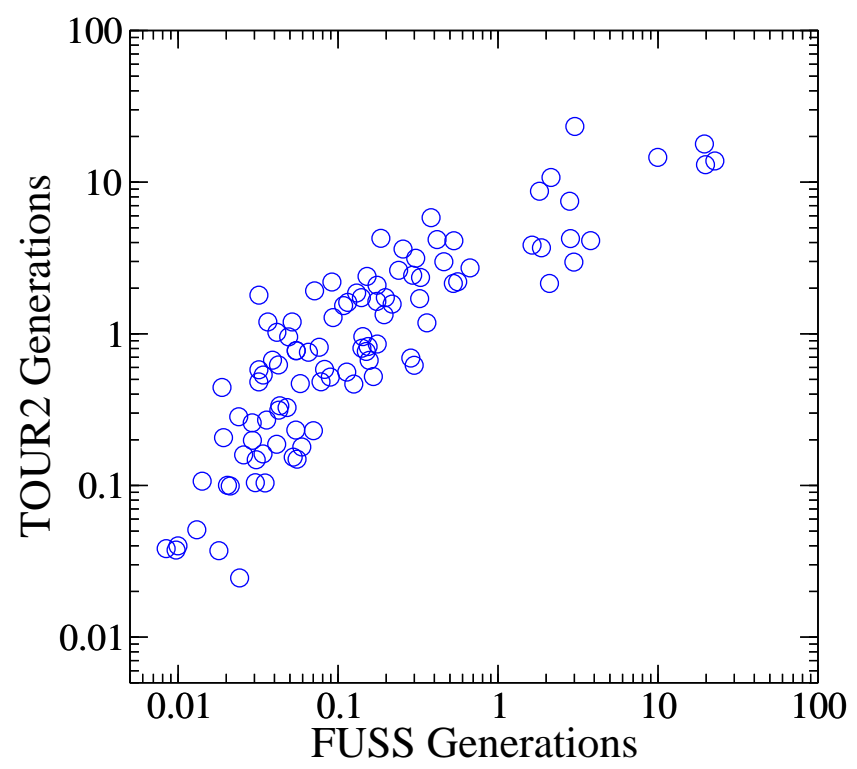

Random Functions

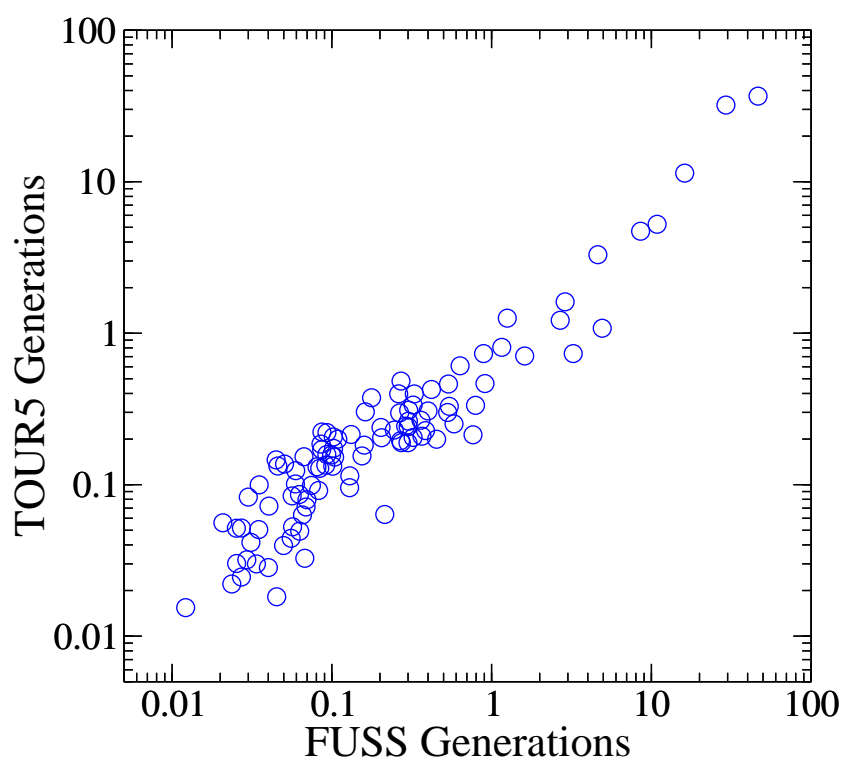

Figure 5: FUSS finds the global maximum typically ten times faster than tournament selection with low selection pressure (left diagram). With increased selection pressure, tournament reaches, but does not pass the performance of FUSS (right diagram).

is sufficient selection pressure for most problems. This performance improvement due to increased selection pressure indicates that tournament selection wasn't becoming significantly stuck in local optima and thus these random function problems where not as deceptive as we had anticipated. We increased selection pressure further by testing TOUR15, but no further performance gains were to be had.

While the strength of FUSS is in dealing with very difficult and deceptive optimization problems, this result demonstrates that even for problems where greater selection pressure is an advantage the performance of FUSS can remain competitive. FUSS also had the advantage that no parameter tuning was required in order to achieve optimum performance for this problem.

\section{Traveling Salesman Problem}

To find the shortest Hamiltonian cycle (path) in a graph of $N$ vertices (cities) connected by edges of certain lengths is a difficult optimization problem. In the following we present preliminary results of a simple evolutionary TSP optimizer with standard selection (here tournament selection) and with FUSS. There are highly specialized (evolutionary) algorithms finding paths less than one percent longer than the optimal path for up to $10^{7}$ cities [7, 8, 9, 10]. Whether FUSS could further improve these algorithms will be studied elsewhere. Here, we are just interested in the performance of FUSS compared to tournament selection on a difficult optimization problem that has real world applications.

The mutation and crossover operators we used were quite simple. Mutation was done by simply switching the position of two of the cities in the solution. For crossover we used the common partial mapped crossover technique [11.

The first test was carried out on a set of TSP problems with random distance matrices. There were 50 TSP problems in total each with 20 cities. The distance between any two cities was chosen uniformly from the interval $[0,1]$. This is a particularly deceptive form of the TSP problem as the usual triangle inequality relation does not hold. For example, the distance between cities $A$ and $B$ might be 0.1 , between cities $B$ and $C 0.2$, and yet the distance between $A$ and $C$ might be 0.8 . The problem still has some structure though as efficient partial solutions tend to be useful building blocks for efficient complete tours. For this test we used a population size of 5,000 and the default mutation and crossover rates of 0.5 . The results appear in Figure 6

We see here that the selection intensity with TOUR2 is too low for the system to converge in a reasonable number of generations. On the other hand the selection intensity under TOUR15 is too high and causes the sys- 


\section{Traveling Salesman Problem - Random}

\section{Traveling Salesman Problem - Sahara}
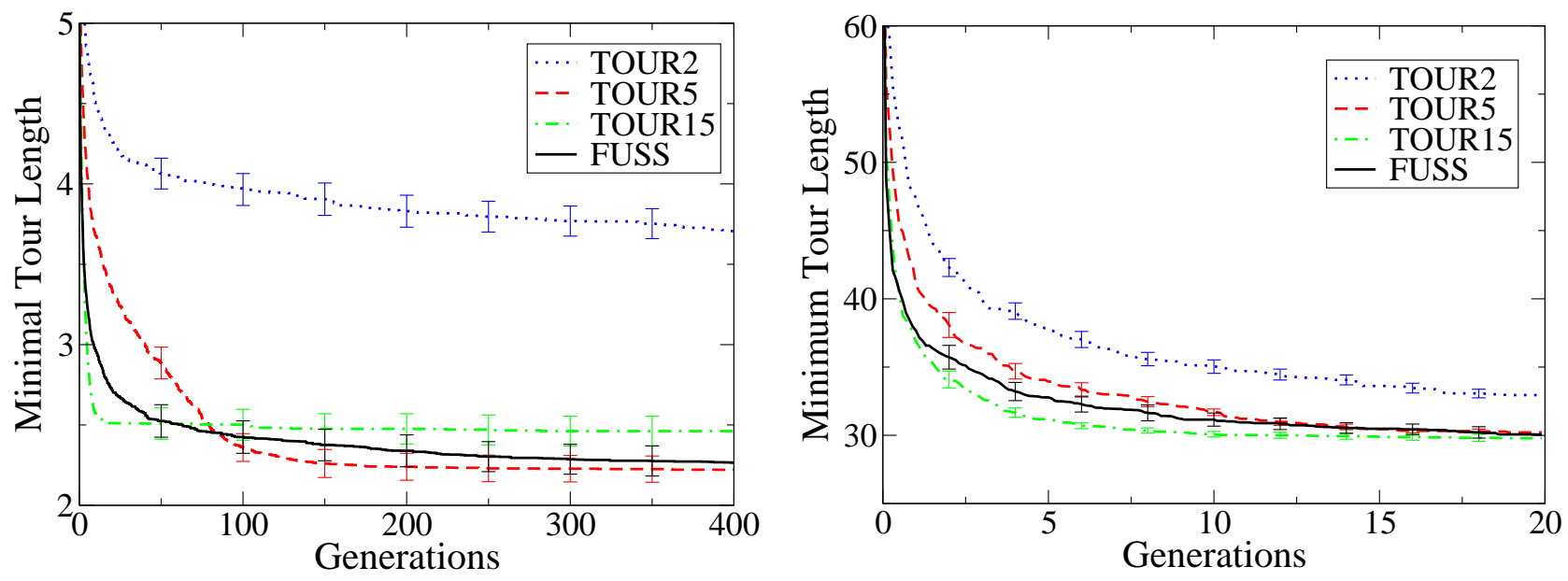

Figure 6: FUSS performs well in both artificial and real TSP problems. For the random TSP problem FUSS converges much faster than TOUR2 but also manages to avoid becoming stuck in a local optimum like TOUR15. In the real TSP problem FUSS again has close to the optimal selection intensity.

tem to become stuck in a local optimum. TOUR5 has about the correct selection intensity for this problem. FUSS outperforms both TOUR2 and TOUR15 and is very close to TOUR5 at the end of the run.

We also tested the system on a number of real TSP problems based on the location of real cities from various countries around the world 12. For these tests the population size was set at 5,000. Based on experimentation we increased the crossover probability to 1.0 and the probability of mutation was reduced to 0.2 for better performance. The results were averaged over a total of 5 runs. The results for the "Sahara" dataset are shown in Figure 6.

Here we see that a higher level of selection intensity is appropriate. FUSS again performs significantly better than TOUR2 and also a little better than TOUR5. At the end of the run FUSS has converged to the same level as both TOUR5 and TOUR15 which is again a positive result for FUSS.

We tested the system on a number of other datasets under various other parameter settings for population size, rate of mutation and crossover etc. and obtained similar results. Nevertheless a fuller analysis comparing other possible mutation and crossover operations and parameters settings will need to be done before more substantive conclusions are possible.

\section{Set Covering Problem}

The set covering problem (SCP) is a reasonably well known NP-complete optimization problem with many real world applications. Let $M \in\{0,1\}^{m \times n}$ be a bi- nary valued matrix and let $c_{j}>0$ for $j \in\{1, \ldots n\}$ be the cost of column $j$. The goal is to find a subset of the columns such that the cost is minimized. Define $x_{j}=1$ if column $j$ is in our solution and 0 otherwise. We can then express the cost of this solution as $\sum_{j=1}^{n} c_{j} x_{j}$ subject to the condition that $\sum_{j=1}^{n} m_{i j} x_{j} \geq 1$ for $i \in\{1, \ldots m\}$.

Our system of representation, mutation operators and crossover follow that used by Beasley 13. We compared the performance of FUSS with tournament selection on a number of standard test problems 14 . For these tests we set the population size to 5,000 , crossover probability to 1.0 , the mutation probability to 0.5 and averaged the performance of the systems over 30 runs on each problem.

The results in Figure 7 were based on the "SCP49" and "SCP41" datasets. Here the performance of FUSS is less impressive. For SCP49 FUSS performs better than TOUR2 however the rate of convergence is still too low. SCP41 is an easy problem with TOUR15 converging in just 4 generations. Nevertheless FUSS is converging very slowly, if at all. It is interesting that FUSS performs poorly on this relatively easy problem when its performance was strong on more difficult problems such as random TSP and the deceptive 2D problem presented earlier. We will look more closely into the reasons for this in the next section. 


\section{Set Covering Problem 49}

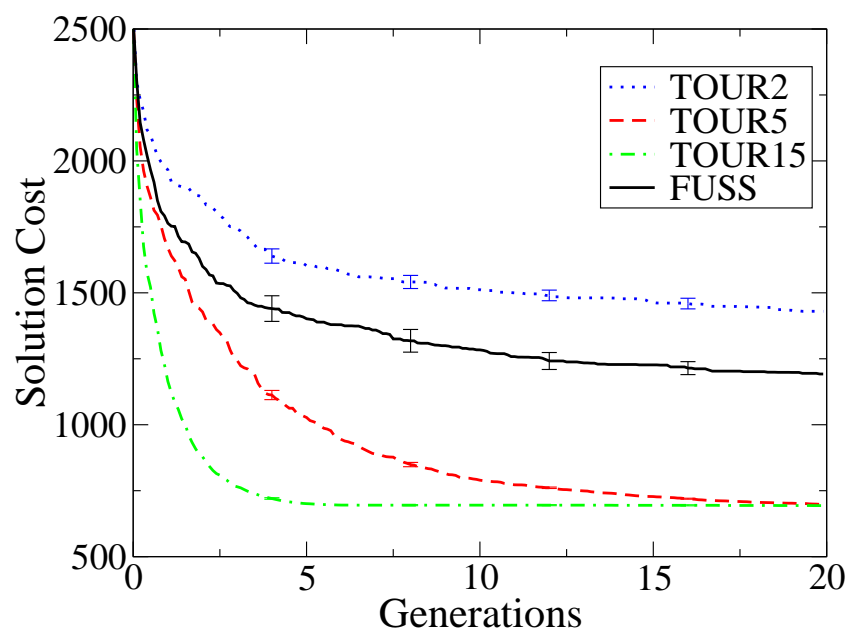

\section{Set Covering Problem 41}

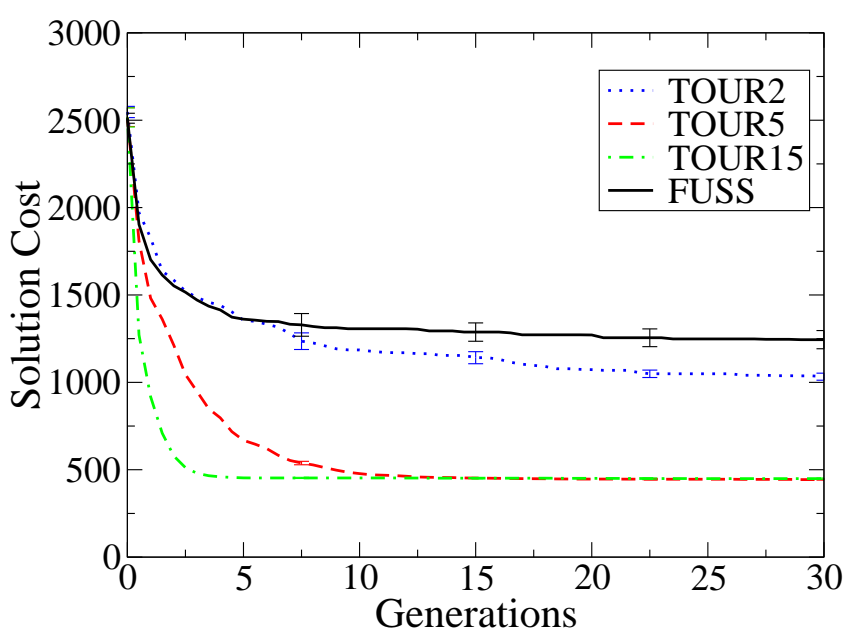

Figure 7: In SCP49 FUSS converges more quickly than TOUR2 but is still too slow. SCP41 is an easy problem as TOUR15 find the optimum very quickly however for some reason FUSS becomes stuck far from the optimum.

\section{Maximum CNF3 SAT}

Maximum CNF3 SAT is a well known NP hard optimization problem [15] that has been extensively studied. A three literal conjunctive normal form (CNF) logical equation is a boolean equation that consists of a conjunction of clauses where each clause contains a disjunction of three literals. So for example, $(a \vee b \vee \neg c) \wedge(a \vee \neg e \vee f)$ is a CNF3 expression. The goal in the maximum CNF3 SAT problem is to find an instantiation of the variables such that the maximum number of clauses evaluate to true. Thus for the above equation if $a=F, b=T, c=T, e=T$, and $f=F$ then just one clause evaluates to true and thus this instantiation gets a score of one. Achieving significant results in this area would be difficult and this is not our aim; we are simply using this problem as a test to compare FUSS and tournament selection.

Our test problems have been taken from the SATLIB collection of SAT benchmark tests [16]. The first test was performed on 30 instances of randomly generated CNF3 forumlae with 150 variables and 645 clauses which are all known to be satisfiable. The second test was performed on 30 instances of "flat" 3 colorable graph coloring problems with 50 vertices and 115 edges which have been expressed in CNF form. The graph coloring problems have a slightly different structure as the clauses contain either 2 or 3 literals.

Our mutation operator simply flips one boolean variable and the crossover operator forms a new individual by randomly selecting for each variable which parent's state to take. The population size was set to 10,000 and the crossover and mutation probabilities were left at the default setting of 0.5. The test was run 30 times for each selection method. The results for both tests appear in Figure 8

In both tests we see that the maximum fitness under FUSS initially climbs very rapidly. Closer inspection showed that it climbs even more rapidly than TOUR15 for the first 0.5 generations. This indicates that FUSS has an extremely high selection intensity to start with, much higher than even TOUR15. After this period FUSS starts to slow down. It appears to become either stuck in a local optimum or the selection intensity falls dramatically, either way, it is then easily passed by the tournament selection schemes. We also tested the system with controlled backbone CNF problems from the same set of benchmark tests and obtained similar results.

We can explain this behavior by considering a simple example. Consider a situation where there is a large number of individuals in a small band of fitness levels, say 10,000 with fitness values ranging from 50 to 70. Add to this population one individual with a fitness value of 73 . Thus the total fitness range is now 24. Whenever FUSS picks a random point from 72 to 73 inclusive this single individual with maximal fitness will be selected. That is, the probability that the single fittest individual will be selected is $2 / 24=0.083$. Now compare this to TOUR15, a selection scheme with high selection intensity. Under TOUR15 the probability that the fittest individual is selected is the same as the probability that it is picked for the sample of 15 elements used for the tournament, that is, $15 / 10000=$ 0.0015 . Thus we can see that in this simple example the probability of selecting the fittest individual under 
CNF3 SAT Benchmark

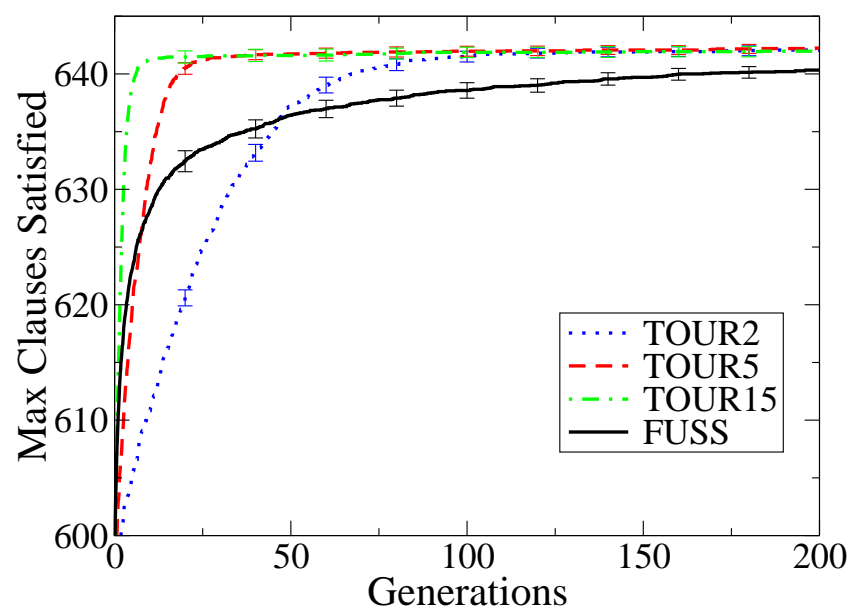

CNF Graph Coloring

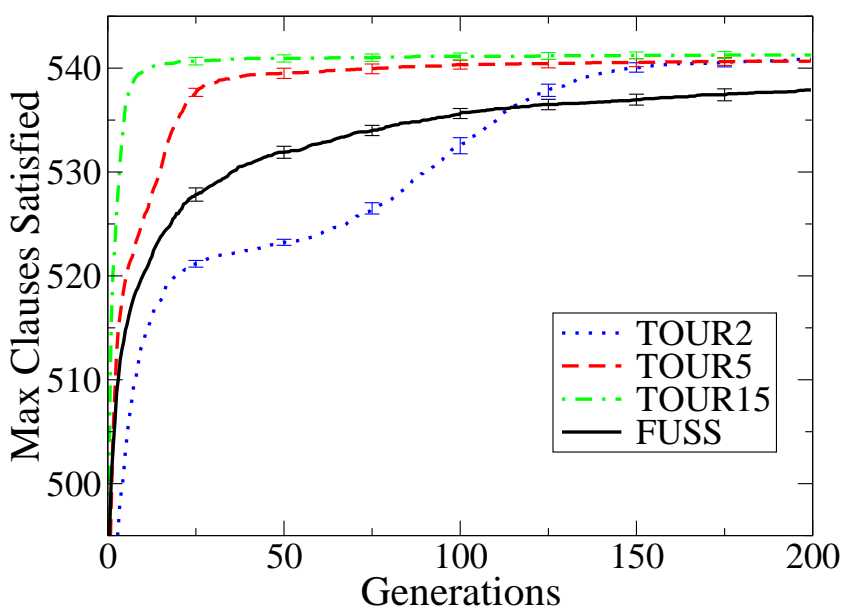

Figure 8: In the CNF3 SAT benchmark with 150 variables and 645 clauses the performance of FUSS was slightly below the performance of tournament selection. The graph coloring problem shows a similar result.

FUSS is over 50 times higher than what it is under TOUR15. This effectively gives FUSS an extremely high selection intensity and would likely result in a very rapidly rising maximal fitness value. If a mutant derived from our highly fit individual had a fitness value higher than 73 then the situation would become much more extreme causing the system to rapidly explore this evolutionary path and fill the higher fitness levels with many highly related individuals in the process.

Once a high level of fitness is reached and further progress becomes difficult the distribution of individuals across the fitness range balances out. When this happens the selection probability for individuals at the highest fitness levels converges towards $\frac{1}{|P|}$ where $|P|$ is the size of the population. Thus the selection intensity becomes very low, much lower than under TOUR15. This explains why FUSS becomes stuck after its initial rapid rise in maximal fitness.

Further experiments have been carried out to test whether these difficulties are responsible for the performance problems we have seen. While FUSS is suited for problems where it is difficult to directly measure and thus control diversity, in the CNF problems we are able measure diversity quite easily by computing hamming distance. Doing so reveals that the diversity in the total population remains very high under FUSS over the evolution of the system, much higher than under the tournament selection schemes. This is what we would expect to see given that FUSS maintains a broad set of both fit and unfit individuals in the population. However if we look at the genetic diversity in the top $10 \%$ of the population we see that diversity under FUSS falls very rapidly and is gener- ally significantly worse than under the tournament selection scheme. Thus while we have succeeded in preserving diversity in the population as a whole, among the fittest individuals in the population diversity is actually rather poor. This is consistent with the scenario described above where FUSS tends to over exploit a very small number of fit individuals in the population.

\section{Conclusions \& Future Re- search Directions}

Theoretical analysis suggests that FUSS should be able to outperform standard selection schemes in some situations, in particular on highly deceptive optimization problems. Our results for a deceptive 2D optimization problem and for TSP problems confirm this. However we have also observed cases where FUSS has performance difficulties. Further analysis indicates that this is due to the greedy nature of FUSS selection in the early stages of the system's evolution. While total genetic diversity was very strong, diversity among the most fit individuals was poor due to the nature of our selection scheme. This suggests that while fitness can be used to control diversity, our current method of doing so is inadequate. We are currently investigating alternates to FUSS which achieve diversity across fitness levels while not exploiting small groups of fit individuals too heavily in the process. Our results so far have been encouraging with diversity being strong both in the population as a whole and among fit individuals. 


\section{References}

[1] D. E. Goldberg and J. Richardson. Genetic algorithms with sharing for multi-modal function optimization. In J. J. Grefenstette, editor, Proceedings of the 2nd International Conference on Genetic Algorithms and their Applications, pages 41-49, Cambridge, MA, July 1987. Lawrence Erlbaum Associates.

[2] K. de Jong. An analysis of the behavior of a class of genetic adaptive systems. Dissertation Abstracts International, 36(10), 5140B, 1975.

[3] R. J. Collins and D. R. Jefferson. Selection in massively parallel genetic algorithms. In R. K. Belew and L. B. Booker, editors, Proceedings of the Fourth International Conference on Genetic Algorithms, San Mateo, CA, 1991. Morgan Kaufmann Publishers.

[4] M. Hutter. Fitness uniform selection to preserve genetic diversity. In X. Yao, editor, Proceedings of the 2002 Congress on Evolutionary Computation (CEC-2002), pages 783-788, Washington D.C, USA, May 2002. IEEE.

[5] M. Hutter. Implementierung eines Klassifizierungs-Systems. Master's thesis, Theoretische Informatik, TU München, 1991. 72 pages with C listing, in German, http://www.idsia.ch/ marcus/ai/pcfs.htm

[6] Alex Rogers and Adam Prügel-Bennett. Modelling the dynamics of a steady-state genetic algorithm. In Wolfgang Banzhaf and Colin Reeves, editors, Foundations of Genetic Algorithms 5, pages 57-68. Morgan Kaufmann, San Francisco, CA, 1999.

[7] S. Lin and B. W. Kernighan. An effective heuristic for the travelling salesman problem. Operations Research, 21:498-516, 1973.

[8] O. Martin and S. Otto. Combining simulated annealing with local search heuristics. Annals of $O p$ erations Research, 63:57-75, 1996.

[9] D. S. Johnson and A. McGeoch. The traveling salesman problem: A case study. In E. H. L. Aarts and J. K. Lenstra, editors, Local Search in Combinatorial Optimization, Discrete Mathematics and Optimization, chapter 8, pages 215-310. WileyInterscience, Chichester, England, 1997.

[10] D. Applegate, W. Cook, and A. Rohe. Chained Lin-Kernighan for large traveling salesman problems. Technical report, Department of Computational and Applied Mathematics, Rice University, Houston, TX, 2000. http://www.isye.gatech.edu/ wcook/papers/ chained_lk.ps.

[11] D. Goldberg and R. Lingle. Alleles. Loci and the traveling salesman problem. In Proceedings of the International Conference on Genetic Algorithms and their Applications, pages 154-159. Lawrence Erlbaum Associates, 1985.

[12] D. Applegate, R. Bixby, V. Chvátal, and W. Cook. National traveling salesman problems. http://www.math.princeton.edu/tsp/world/ countries.html, 2003.

[13] J. Beasley and P. Chu. A genetic algorithm for the set covering problem. European Journal of Operational Research, 94:392-404, 1996.

[14] J. Beasley. Or-library. http://mscmga.ms.ic.ac.uk/jeb/orlib/scpinfo.html 2003.

[15] P. Crescenzi and V. Kann. A compendium of NP optimization problems. http://www.nada.kth.se/ viggo/problemlist/ compendium.html, 2003.

[16] Holger H. Hoos and Thomas Stützle. SATLIB: An Online Resource for Research on SAT. In T. Walsh I.P.Gent, H.v.Maaren, editor, SAT 2000, pages 283-292. IOS press, 2000. 